\title{
ASKAP and MeerKAT surveys of the Magellanic Clouds
}

\author{
Jacco Th. van Loon \\ Lennard-Jones Laboratories, Keele University, ST5 5BG, United Kingdom \\ E-mail: jacco@astro.keele.ac.uk
}

\section{The GASKAP Team}

Hector Arce, Amanda Bailey, Indra Bains, Ayesha Begum, Kenji Bekki, Nadya Ben Bekhti, Joss Bland-Hawthorn, Kate Brooks, Chris Brunt, Michael Burton, James Caswell, Maria Cunningham, John Dickey, Kevin Douglas, Simon Ellingsen, Jayanne English, Robert Estalella, Alyson Ford, Tyler Foster, Bryan Gaensler, Jay Gallagher, Steven Gibson, José Girart, Paul Goldsmith, José F. Gómez, Yolanda Gómez, Anne Green, James Green, Matt Haffner, Carl Heiles, Fabian Heitsch, Patrick Hennebelle, Tomoya Hirota, Melvin Hoare, Hiroshi Imai, Hideyuki Izumiura, Gilles Joncas, Paul Jones, Peter Kalberla, Ji-hyun Kang, Akiko Kawamura, Jürgen Kerp, Charles Kerton, Bon-Chul Koo, Roland Kothes, Stan Kurtz, Maša Lakićević, Tom Landecker, Alex Lazarian, Nadia Lo, Felix Lockman, Loris Magnani, Naomi McClure-Griffiths, Karl Menten, Victor Migenes, Marc-Antoine Miville-Deschênes, Erik Muller, Akiharu Nakagawa, Hiroyuki Nakanishi, Jun-ichi Nakashima, David Nidever, Lou Nigra, Josh Peek, Miguel Pérez-Torres, Chris Phillips, Mary Putman, Anthony Remijan, Philipp Richter, Peter Schilke, Yoshiaki Sofue, Snežana Stanimirović, Daniel Tafoya, Russ Taylor, Wen-Wu Tian, Lucero Uscanga, Jacco van Loon, Maxim Voronkov, Bart Wakker, Andrew Walsh, Tobias Westmeier, Benjamin Winkel, Ellen Zweibel

\section{The MagiKAT Team}

Gemma Bagheri, Amanda Bailey, Sudhanshu Barway, Yuri Beletsky, Nadya Ben Bekhti, Jean-Philippe Bernard, Michael Bietenholz, Roy Booth, Caroline Bot, Elias Brinks, Maria-Rosa Cioni, Maria Cunningham, Erwin de Blok, John Dickey, Kevin Douglas, Chris Evans, Brad Gibson, Steven Gibson, Sharmila Goedhart, José Gómez, Karl Gordon, Marijke Haverkorn, Fabian Heitsch, Patrick Hennebelle, Benne Holwerda, Remy Indebetouw, Frank Israel, Paul Jones, Peter Kalberla, Akiko Kawamura, Jürgen Kerp, Bärbel Koribalski, Mark Krumholz, Maša Lakićević, Nadia Lo, Margaret Meixner, Karl Menten, Erik Muller, Joana Oliveira, Peter Sarre, Marta Sewiło, Lisette Sibbons, Keith Smith, Jacco van Loon, Wouter Vlemmings, Fabian Walter, Patricia Whitelock, Benjamin Winkel, Albert Zijlstra 
The Magellanic Clouds are a stepping stone from the overwhelming detail of the Milky Way in which we are immersed, to the global characteristics of galaxies both in the nearby and distant universe. They are interacting, gas-rich dwarf galaxies of sub-solar metallicity, not unlike the building blocks that assembled the large galaxies that dominate groups and clusters, and representative of the conditions at the height of cosmic star formation. The Square Kilometre Array (SKA) can make huge strides in understanding galactic metabolism and the ecological processes that govern star formation, by observations of the Magellanic Clouds and other, nearby Magellanictype irregular galaxies. Two programmes with SKA Pathfinders attempt to pave the way: the approved Galactic ASKAP Spectral Line Survey (GASKAP) includes a deep survey in H I and $\mathrm{OH}$ of the Magellanic Clouds, whilst MagiKAT is proposed to perform more detailed studies of selected regions within the Magellanic Clouds - also including Faraday rotation measurements and observations at higher frequencies. These surveys also close the gap with the revolutionizing surveys at far-IR wavelengths with the Spitzer Space Telescope and Herschel Space Observatory.

ISKAF2010 Science Meeting - ISKAF2010

June 10-14, 2010

Assen, the Netherlands 


\section{The Magellanic Clouds: cosmic evolution up close}

We are incredibly fortuitous to find ourselves in the position to study two rather substantial actively star-forming dwarf galaxies right on our doorstep $(\approx 50 \mathrm{kpc}$ for the Large Magellanic Cloud and $\approx 60 \mathrm{kpc}$ for the Small Magellanic Cloud), through a window of transparency. They hold the keys to unlocking many of the secrets pertaining to the way the Universe has evolved from what was once a dark place to one of brilliance and activity. Much of the physical processes at play, within and between galaxies of the Magellanic type but which are - or have been - prevalent in other types of galactic environment as well, are accessible to the sensitive and sharp radio eyes of the upcoming pathfinders for the Square Kilometre Array (SKA).

In the cold dark matter hierarchical galaxy assembly paradigm, the young and dense Universe was teeming with gas clouds whizzing around within the gravitational lure of nascent clusters of galaxies, arising from fluctuations in the dark matter distribution. Colliding and merging to form the larger spiral and elliptical galaxies that are today's Universe's lighthouses, many of these building blocks have avoided this destiny and are now still the most plentiful dark-matter dominated systems. Most have since consumed their gas by converting it into stars and by stripping - be it by external processes (galaxy interaction) or by internal processes (winds and supernovae). The more massive or more isolated among them have been able to retain enough gas to sustain star formation over prolonged periods of time right till the present day, leading to the buildup of chemical richesse (the LMC's gas has a metal content $\approx 1 / 2$ that of the Sun, and the SMC $\approx 1 / 5$ ). These gas-rich dwarf galaxies are both tantalising relics of the Universe's re-birth during the epoch of cosmic re-ionization, as well as fascinating miniature galaxies in their own right.

The Magellanic Clouds are a splendid showcase of galaxy interaction. The SMC's reservoir of gas has been depleted by the pull from the significantly more massive LMC, forming the Magellanic Bridge that connects the two - star formation is occurring in the denser portions of this tidal feature. Together, the Magellanic Clouds tumble through the Milky Way's Halo - which extends at least twice as far as where the Magellanic Clouds presently are - causing loosely-bound gas to feed a tail that spans half the celestial hemisphere: the Magellanic Stream. While the tidal forces exerted by the Galaxy become more effective further down-stream, the ram pressure exerted by the hot Halo gas on the speedy Magellanic Clouds is tremendous: it has caused the head-tail morphology of the Clouds-Stream and likely compressed gas at the frontal rim of the LMC which led to the vigorous formation of stars including the Tarantula Nebula "mini-starburst". Though we probably witness the Magellanic Clouds during an unusual phase - their large proper motions cast doubt on them being bound to the Galaxy (Besla et al. 2006) and the SMC is still very gas-rich this is probably not their first (nor last) close encounter with each other and with the Galaxy.

\section{The Magellanic Clouds: astrophysical laboratories}

The Magellanic Clouds have historically been of great significance for many fields of astrophysics, thanks largely to their known distance and their proximity. From the discovery of the period-luminosity relation of Cepheid variable stars by Henrietta Leavitt in 1908, which set the fundamental distance scale of the Universe, to the progenitor with the wrong colour of supernova 1987A, the Magellanic Clouds are full of surprises that turn long-held beliefs up-side down. 
The Magellanic Clouds offer a spectacular manifestation of the multi-phase interstellar medium (ISM) (Chu 2009): shells produced by fast stellar winds and supernovae give the warm neutral medium the appearance of bubble-foam; these are filled with hot plasma, but they are bound to succomb to disruptive processes including rotational shear, shell-shell collisions, and thermal instabilities within the shell walls. The dissipation of energy causes a trickle down the turbulent cascade towards smaller scales, where other sources await to inject energy and momentum. A question remaining concerns the importance of magnetic fields in regulating the ISM dynamics.

One of the mysteries in star formation lies at its base: the transformation of the warm ISM into molecular clouds, and the subsequent collapse of cores on their way to forming stars. The commonly used tracer of molecular clouds, carbon-monoxide is detected only in the densest parts, leaving much of the $\mathrm{H}_{2}$ cloud envelopes unseen - this may be traced by cold $\mathrm{H}$ I or OH. Given that the contraction of cores to the point of nuclear fusion has to pass several hurdles via cooling by metallic material and via diffusion of the lightly-ionized gas through an intensified magnetic field, one might expect the outcome of this process to depend on metallicity (Oliveira 2009). Once massive protostars are formed, their outflows and ionizing radiation act upon the gas surrounding them. Protostars are found at far-IR wavelengths (Sewiło et al. 2010), by masers, and in later stages as ultra-compact $\mathrm{H}$ II regions which shine brightly through free-free emission at radio wavelengths.

Stars in their final stages lose a significant fraction of their mass in the form of a stellar wind fast for hot stars, slow for cool stars. In the winds from red (super)giants (except carbon stars), $\mathrm{OH}$ maser emission offers the best way to measure the wind speed, which is connected to the luminosity and to the gas:dust ratio and hence metallicity; tests in the Magellanic Clouds are thus extremely useful (Marshall et al. 2008). When supernovae and their remnants expand into the surrounding ISM, they collect matter and magnetic field from it (e.g., Otsuka et al. 2010). If they encounter a molecular cloud they may disrupt it or induce it to collapse - the outcome is not yet clear.

\section{Radio surveys of the Magellanic Clouds: a revolution in the making}

The most comprehensive view of the atomic gas in the Magellanic Clouds is had through the H I surveys with the Australia Telescope Compact Array and Parkes dish (LMC: Kim et al. 2003; SMC: Stanimirović et al. 1999). These have an angular resolution of $1^{\prime}$ (LMC) and 1.6' (SMC), a velocity resolution of $1.6 \mathrm{~km} \mathrm{~s}^{-1}$, and reach brightness sensitivities of $\sim 2 \mathrm{~K}$. Impressive though they are, they are limited in their ability to detect cold gas. H I absorption surveys have detected such gas in a small number of sightlines through the LMC (Marx-Zimmer et al. 2000) and SMC (Dickey et al. 2000). Likewise, Faraday rotation measurements have been made in several directions suggesting an ordered global field (LMC: Gaensler et al. 2005; SMC: Mao et al. 2008). A much denser grid of measurements is needed to establish the origin of the field and the rôle it plays at the scales of star-forming regions in the Magellanic Clouds $\left(1-10^{\prime}\right)$.

Radio continuum emission traces free electrons via free-free emission or synchrotron emission if gyrating within a magnetic field. Surveys of the Magellanic Clouds have been conducted at frequencies between 1.4-8.6 GHz (LMC: Dickel et al. 2005; Hughes et al. 2007; SMC: Filipović et al. 2002) at scales between 20-40" - smaller for bright sources such as supernova remnants.

Masers have been detected in the LMC from protostars (Ellingsen et al. 2010; $c f$. Oliveira et al. 2006) and evolved stars (Marshall et al. 2004; $c f$. van Loon et al. 2001), but not in the SMC. 
Whilst existing H I surveys are a good match to the far-IR resolution of IRAS, modern IR surveys have progressed to much greater detail: around 6-40" at 24-160 $\mu \mathrm{m}$ with the Spitzer Space Telescope and a similar range but at 60-600 $\mu \mathrm{m}$ with the Herschel Space Observatory (Meixner et al. 2010). This means that currently the resolution of pseudo- $\mathrm{H}_{2}$ maps that can be derived from the combination of $\mathrm{H}$ I and far-IR data is (severely) limited by that of the H I data.

Planned and proposed surveys of the Magellanic Clouds with Southern SKA pathfinders, to start in 2013, GASKAP and MagiKAT will be deeper and sharper in both an angular and kinematic sense, by an order of magnitude or more over existing surveys (Table 1).

\begin{tabular}{|l|l|l|}
\hline Survey & Tracer & Particulars \\
\hline GASKAP & $\begin{array}{l}\text { H I emission/absorption } \\
\text { OH emission/absorption }\end{array}$ & $\begin{array}{l}S_{\mathrm{rms}}=0.76 \mathrm{~K} \text { at } 20^{\prime \prime}, 1 \mathrm{~km} \mathrm{~s}^{-1}\left(\text { also } 8^{\prime \prime}, 0.2 \mathrm{~km} \mathrm{~s}^{-1}\right) \\
\text { few mJy }(5 \sigma), \text { at } 1612+1665+1667 \mathrm{MHz}\end{array}$ \\
\hline MagiKAT & $\begin{array}{l}\text { H I emission/absorption } \\
\text { OH emission/absorption } \\
\mathrm{CH}_{3} \mathrm{OH} \text { masers } \\
\text { high-frequency continuum } \\
\text { Faraday rotation }\end{array}$ & $\begin{array}{l}S_{\mathrm{rms}}=0.49 \mathrm{~K} \text { at } 20^{\prime \prime}, 1 \mathrm{~km} \mathrm{~s}^{-1}\left(\text { also } 8^{\prime \prime}, 0.2 \mathrm{~km} \mathrm{~s}^{-1}\right) \\
\text { few my }(5 \sigma), \text { at } 1612+1665+1667+1720 \mathrm{MHz} \\
\text { at } 12.2 \mathrm{GHz}, \text { few mJy }(5 \sigma), \text { few pointings per } 1^{\circ} \text { field } \\
\text { at } 8-14 \mathrm{GHz}, \text { full Stokes, } \sim 1^{\prime \prime} \text { resolution }\end{array}$ \\
at $0.6-2.5 \mathrm{GHz}, \sim 1000$ measurements per $1^{\circ}$ field \\
\hline
\end{tabular}

Table 1: Summary of the approved GASKAP and proposed MagiKAT surveys of the Magellanic Clouds.

\subsection{GASKAP}

The Galactic ASKAP Spectral Line Survey (GASKAP) is an approved Survey Science Project at the Australian SKA Pathfinder (ASKAP). It includes the Magellanic Clouds among its deepest survey components (yet a modest $627 \mathrm{hr}$ ) in $\mathrm{H} \mathrm{I}$ and $\mathrm{OH} \mathrm{1612,} 1665$ and $1667 \mathrm{MHz}$. The resolution is nominally $20^{\prime \prime}$ but postage-stamp cubes are planned for the brightest compact sources at $8^{\prime \prime}$.
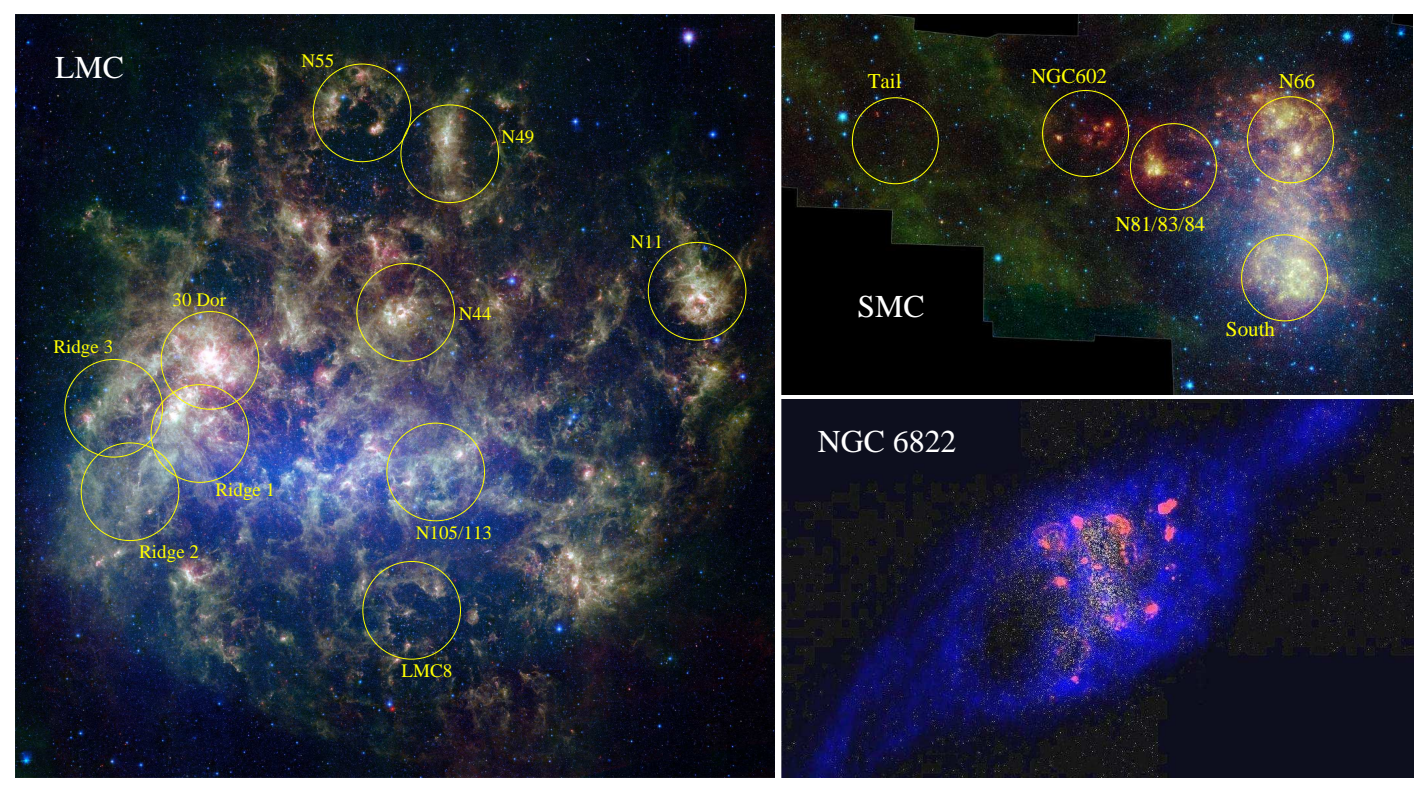

Figure 1: Proposed target fields for MagiKAT — circles are for 1.4-GHz $\left(1^{\circ}\right), \mathrm{NGC} 682$ is covered entirely. 


\subsection{MagiKAT}

MagiKAT is a proposed Large Project for the South African SKA pathfinder, MeerKAT. It specifically targets the Magellanic Clouds $(\approx 4400 \mathrm{hr}$, plus the next-nearest Magellanic-type galaxy, NGC 6822), but only in ten selected fields in the LMC and five in the SMC, each $1^{\circ}$ in diameter at $1.4 \mathrm{GHz}$ (Fig. 1). Besides $\mathrm{H}$ I and all four $\mathrm{OH}$ lines it also measures the continuum at higher and searches for methanol masers at $12.2 \mathrm{GHz}$ - in correspondingly smaller regions within the $1^{\circ}$ fields - and it will obtain Faraday rotation measurements of the magnetic field by observing background radio galaxies between $0.6-2.5 \mathrm{GHz}$. The expectation is that over $10^{4}$ Faraday rotation measurements will be performed, along with hundreds $\mathrm{H}$ I absorption measurements.

\section{Gearing up for the future}

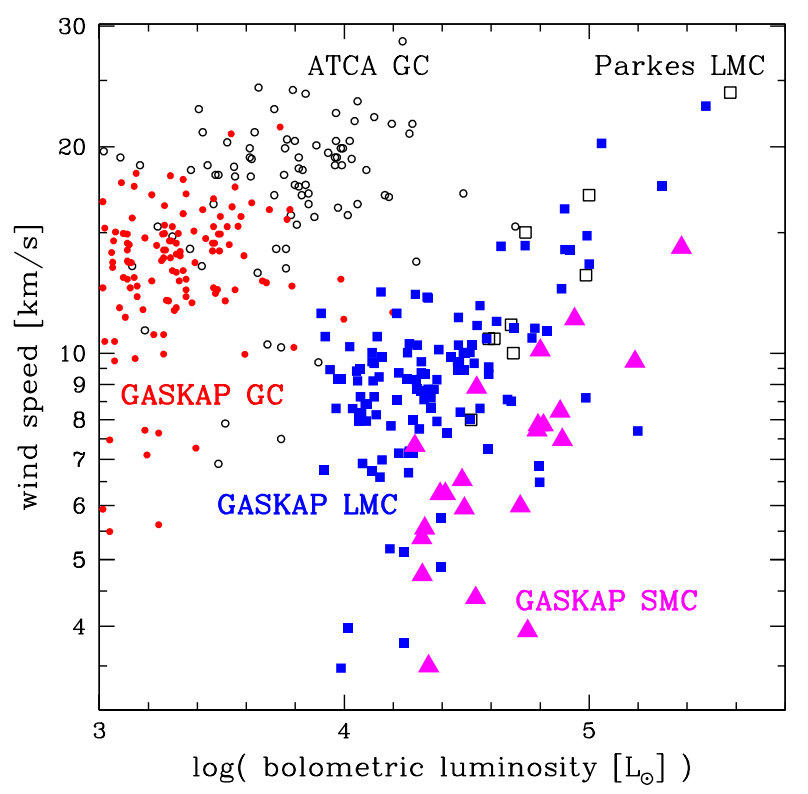

Figure 2: Prediction for the yield of $\mathrm{OH} 1612-\mathrm{MHz}$ masers from evolved stars in the GASKAP survey.

GASKAP has entered the design phase. As part of this endeavour, it has just completed an $\mathrm{H} \mathrm{I}$ simulation through the ASKAP simulator and pipeline on scaled data from the Galactic All-Sky Survey (GASS). It is now finalising preparations for a realistic $\mathrm{OH}$ maser simulation, improving upon the scaling of known Galactic populations (Fig. 2). These simulations are used to assess data reduction requirements, and to test source finding algorithms; in the case of the $\mathrm{OH}$ maser simulations these can be compared with real observations with ASKAP (and MeerKAT) to test the validity of the underlying assumptions regarding the $\mathrm{OH}$ maser populations.

Exciting times lie ahead for these two superb pathfinders on the road to unlocking the secrets of the Magellanic Clouds, one giant step towards understanding the workings of the Universe. They will also demonstrate what further can be gained from using the SKA for studies of diffuse matter.

\section{Acknowledgments}

The author cherishes happy memories of a lovely time with charming housemates. 


\section{References}

[1] G. Besla, N. Kallivayalil, L. Hernquist, B. Robertson, T.J. Cox, R.P. van der Marel, C. Alcock, Are the Magellanic Clouds on their first passage about the Milky Way?, 2007, ApJ 668, 949 [astro-ph/0703196].

[2] Y.-H. Chu, The violent interstellar medium of the Magellanic Cloud System, in proceedings of The Magellanic System: Stars, Gas, and Galaxies, IAUS 256, 166.

[3] J.R. Dickel, V.J. McIntyre, R.A. Gruendl, D.K. Milne, A 4.8 and 8.6 GHz survey of the Large Magellanic Cloud, 2005, AJ 129, 790 [astro-ph/ 0410683 ].

[4] J.M. Dickey, U. Mebold, S. Stanimirović, L. Staveley-Smith, Cold atomic gas in the Small Magellanic Cloud, 2000, ApJ 536, 756.

[5] S.P. Ellingsen, S.L. Breen, J.L. Caswell, L.J. Quinn, G.A. Fuller, Masers associated with high-mass star formation regions in the LMC, 2010, MNRAS 404, 779 [arXiv: 1001 . 2169].

[6] M. Filipović, T. Bohlsen, W. Reid, L. Staveley-Smith, P.A. Jones, K. Nohejl, G. Goldstein, An ATCA radio continuum study of the Small Magellanic Cloud, 2002, MNRAS 335, 1085.

[7] B.M. Gaensler, M. Haverkorn, L. Staveley-Smith, J.M. Dickey, N.M. McClure-Griffiths, J.R. Dickel, M. Wolleben, The magnetic field of the Large Magellanic Cloud revealed through Faraday rotation, 2005, Sci 307, 1610 [astro-ph/ 0503226 ].

[8] A. Hughes, L. Staveley-Smith, S. Kim, M. Wolleben, M. Filipović, An Australia Telescope Compact Array 20-cm radio continuum study of the LMC, 2007, MNRAS 382, 543 [arXiv: 0709. 1990].

[9] S. Kim, L. Staveley-Smith, M.A. Dopita, R.J. Sault, K.C. Freeman, Y. Lee, Y.-H. Chu, A neutral hydrogen survey of the Large Magellanic Cloud, 2003, ApJS 148, 473.

[10] S.A. Mao, B.M. Gaensler, S. Stanimirović, M. Haverkorn, N.M. McClure-Griffiths, L. Staveley-Smith, J.M. Dickey, A radio and optical polarization study of the magnetic field in the Small Magellanic Cloud, 2008, ApJ 688, 1029 [arXiv: 0807 . 1532].

[11] J.R. Marshall, J.Th. van Loon, M. Matsuura, P.R. Wood, A.A. Zijlstra, P.A. Whitelock, Asymptotic giant branch superwind speed at low metallicity, 2004, MNRAS 355, 1348 [astro-ph / 0410120 ].

[12] M. Marx-Zimmer, U. Herbstmeier, J.M. Dickey, F. Zimmer, L. Staveley-Smith, U. Mebold, A study of cool gas in the Large Magellanic Cloud, 2000, A\&A 354, 787.

[13] M. Meixner, et al., HERITAGE, 2010, A\&A 518, L71 [arXiv: 1006 . 0985].

[14] J.M. Oliveira, The star formation process in the Magellanic Clouds, 2009, in proceedings of The Magellanic System: Stars, Gas, and Galaxies, IAUS 256, 191 [arXiv: 0812 . 2360].

[15] J.M. Oliveira, J.Th. van Loon, S. Stanimirović, A.A. Zijlstra, Massive young stellar objects in the Large Magellanic Cloud, 2006, MNRAS 372, 1509 [ast ro-ph/ 0609036 ].

[16] M. Otsuka, et al., Dust in the bright supernova remnant $N 49$ in the LMC, 2010, A\&A 518, L139 [arXiv:1005.2787].

[17] M. Sewiło, et al., The youngest massive protostars in the Large Magellanic Cloud, 2010, A\&A 518, L73 [arXiv: 1005.2592$]$.

[18] S. Stanimirović, L. Staveley-Smith, J.M. Dickey, R.J. Sault, S.L. Snowden, The large-scale H I structure of the Small Magellanic Cloud, 1999, MNRAS 302, 417.

[19] J.Th. van Loon, A.A. Zijlstra, V. Bujarrabal, L.- $\AA$. Nyman, Circumstellar masers in the Magellanic Clouds, 2001, A\&A 368, 950 [astro-ph/ 0101125 ]. 